\title{
Penalization schemes for reflecting stochastic differential equations
}

\author{
ROGER PETTERSSON \\ Department of Mathematical Statistics, Box 118, Lund University, 22100 Lund, Sweden
}

We consider discrete penalization schemes for reflecting stochastic differential equations. The convergence results obtained by Liu are generalized and refined. We also compare the penalization schemes with a more well-known recursive projection scheme.

Keywords: penalization schemes; reflections; stochastic differential equations

\section{Introduction}

A solution to a reflecting stochastic differential equation (RSDE) is a diffusion process constrained to a given set, in our case with normal reflection at the boundary. There are a variety of different applications of RSDEs (see, for example, Asmussen 1992, Krée and Soize 1986, Chapter XIV and Shepp and Shiryaev 1994) where a suitable numerical scheme is of importance.

Lions et al. (1993) and Menaldi (1983) constructed solutions of RSDEs by considering diffusion processes which are 'penalized' by a term $\beta_{\lambda}(x)=\{x-\Pi(x)\} / \lambda$, where $\Pi$ denotes projection onto the constraining set. As $\lambda \downarrow 0$, convergence towards a solution of the RSDE was obtained. These results were recently recovered by Storm (1995).

Liu (1993) showed convergence of Euler approximations for the penalizing stochastic differential equations (SDEs). For a small but fixed step size $\Delta t, \lambda$ was chosen to be equal to $(\Delta t)^{1 / 2}$. The main idea in this paper is to consider convergence for all choices of $\lambda \geqslant \Delta t$. We obtain that a suitable choice of $\lambda \geqslant \Delta t$ seems to be $\lambda=\Delta t$. When $\lambda<\Delta t$, the penalty term tends to push the approximating sequence inwards too much for the constraining set to be useful.

We also compare the penalization scheme with the 'projection scheme' investigated by, for example, Słomiński (1994) and Pettersson (1995).

\section{Notation and recalled results}

Assume that $\odot$ is an open, convex and bounded set in $\mathfrak{2}^{d}$. Denote by $\Pi$ the projection map onto $\bar{\odot}$ : 


$$
\Pi(x)=\arg \min _{y \in \overline{\mathscr{C}}}|x-y|, \quad x \in \mathfrak{2}^{d},
$$

where $|\cdot|$ is the usual Euclidean norm. Let

$$
\beta(x)=x-\Pi(x), \quad \beta_{\lambda}(x)=\frac{\beta(x)}{\lambda}, \quad x \in \mathfrak{\Re}^{d} .
$$

Let $x_{0}$ be a given point in $\mathscr{Q}$. From the work of Menaldi (1983) there exists some $\gamma>0$ such that

$$
\gamma|\beta(x)| \leqslant\left\langle x-x_{0}, \beta(x)\right\rangle, \quad \forall x \in \mathfrak{\Re}^{d},
$$

where $\langle\cdot, \cdot\rangle$ is the usual inner product. We shall also use the facts that

$$
\begin{gathered}
|\beta(x)-\beta(y)| \leqslant|x-y|, \quad \forall x, \forall y \in \mathfrak{R}^{d}, \\
-\left\langle x-y, \beta_{\lambda}(x)\right\rangle \leqslant-\lambda\left|\beta_{\lambda}(x)\right|^{2}, \quad \forall x \in \mathfrak{A}^{d}, \forall y \in \overline{\mathscr{O}}, \forall \lambda>0,
\end{gathered}
$$

(see, for example, Aubin and Cellina 1984, pp. 24 and 33).

Let $(\Omega, \mathscr{F}, P)$ be a probability space with filtration $\left\{\mathscr{F}_{t}\right\}_{t \geqslant 0}$ satisfying the usual conditions. Let $\{B(t)\}_{t \geqslant 0}$ be an $m$-dimensional $\left\{\mathscr{F}_{t}\right\}$-adapted Brownian motion. Assume that $b: \mathfrak{l}^{d} \mapsto \mathfrak{R}^{d}$ and $\sigma: \mathfrak{l}^{d} \mapsto \mathfrak{R}^{d} \times \mathfrak{R}^{m}$ are Lipschitz continuous normed by $|b|=$ $\left(\sum_{i=1}^{d} b_{i}^{2}\right)^{1 / 2}$ and $|\sigma|=\left(\sum_{i=1, j=1}^{d, m} \sigma_{i j}^{2}\right)^{1 / 2}$. For cadlags $z$ let $\|z\|_{t}=\sup \{|z(t)|: 0 \leqslant t \leqslant T\}$. Let $0<T<\infty$. Let $c$ be a generic constant, i.e., the value of $c$ may change from line to line.

We consider the RSDE

$$
\mathrm{d} \xi(t)=b(\xi(t)) \mathrm{d} t+\sigma(\xi(t)) \mathrm{d} B(t)-\mathrm{d} \eta(t), \quad \xi(0)=x_{0} .
$$

Definition 2.1. A couple $(\xi, \eta)$ is said to be a solution of the RSDE (4) on $[0, T]$ if

(i) $\xi$ and $\eta$ are continuous and progressively measurable,

(ii) $\xi(t) \in \mathscr{O} \forall t$ and $\eta$ has bounded variation on $[0, T]$,

(iii) for all continuous and progressively measurable processes $v$ taking values in $\overline{\mathscr{O}}$,

$$
\int_{0}^{t}\langle\xi(s)-v(s), \mathrm{d} \eta(s)\rangle \geqslant 0,
$$

and $\xi(t)=x_{0}+\int_{0}^{t} b(\xi(s)) \mathrm{d} s+\int_{0}^{t} \sigma(\xi(s)) \mathrm{d} B(s)-\eta(t)$.

By the Lipschitz conditions of $b$ and $\sigma$ and (2), there exists, for each $\lambda>0$, a unique solution $\xi_{\lambda}$ to the $\mathrm{SDE}$

$$
\mathrm{d} \xi_{\lambda}(t)=b\left(\xi_{\lambda}(t)\right) \mathrm{d} t+\sigma\left(\xi_{\lambda}(t)\right) \mathrm{d} B(t)-\beta_{\lambda}\left(\xi_{\lambda}(t)\right) \mathrm{d} t, \quad \xi_{\lambda}(0)=x_{0} .
$$

Menaldi (1983) showed that there exists a unique solution to the RSDE (4). Furthermore, according to Menaldi (1983, Remark 3.1) and the arguments of Liu (1993), we obtain the following result:

$$
\mathrm{E}\left\|\xi_{\lambda}-\xi\right\|_{T}^{2} \leqslant c \lambda^{1-\alpha}, \quad \forall \alpha>0
$$




\section{Euler approximations of $\xi_{\lambda}$}

Liu (1993) considered Euler approximations $\xi_{\lambda}^{\Delta t}$ for (5):

$$
\xi_{\lambda}^{\Delta t}\left(t_{k}\right)=\xi_{\lambda}^{\Delta t}\left(t_{k-1}\right)+b\left(\xi_{\lambda}^{\Delta t}\left(t_{k-1}\right)\right) \Delta t_{k}+\sigma\left(\xi_{\lambda}^{\Delta t}\left(t_{k-1}\right)\right) \Delta B_{k}-\beta_{\lambda}\left(\xi_{\lambda}^{\Delta t}\left(t_{k-1}\right)\right) \Delta t_{k},
$$

where, for some integer $n, 0=t_{0}<t_{1}<\cdots<t_{n}=T, \Delta t_{k}=t_{k}-t_{k-1}=\Delta t$ and $\Delta B_{k}=$ $B\left(t_{k}\right)-B\left(t_{k-1}\right)$. For $\quad t \in\left[t_{k-1}, t_{k}\right)$, let $\quad \xi_{\lambda}^{\Delta t}(t)=\xi_{\lambda}^{\Delta t}\left(t_{k-1}\right)+b\left(\xi_{\lambda}^{\Delta t}\left(t_{k-1}\right)\right)\left(t-t_{k-1}\right)+$ $\sigma\left(\xi_{\lambda}^{\Delta t}\left(t_{k-1}\right)\right)\left\{B(t)-B\left(t_{k-1}\right)\right\}$. Liu obtained that

$$
\mathrm{E}\left|\xi_{\lambda}^{\Delta t}(T)-\xi_{\lambda}(T)\right|^{2}=\mathrm{O}\left(\Delta t^{1 / 2-\alpha}\right), \quad \forall \alpha \in\left(0, \frac{1}{2}\right)
$$

if $\Delta t=\lambda^{2}$ is small.

We show a refined and generalized result of (8). By the expression ' $\sigma$ is bounded' we mean that, for some constant $c>0,|\sigma(x)| \leqslant c$ for all $x \in \mathfrak{R}^{d}$.

Theorem 3.1. Assume that $b$ and $\sigma$ are Lipschitz continuous and $\sigma$ is bounded. Then

$$
\mathrm{E}\left\|\xi_{\lambda}^{\Delta t}-\xi_{\lambda}\right\|_{T}^{2}=\mathrm{O}\left[\left\{\Delta t \log \left(\frac{1}{\Delta t}\right)\right\}^{1 / 2}\right]
$$

if $\Delta t \leqslant \lambda$ and $\lambda$ is small.

By (6) and Theorem 3.1 it follows that

$$
\exists c>0, \quad \mathrm{E}\left\|\xi_{\lambda}^{\Delta t}-\xi\right\|_{T}^{2} \leqslant c\left[\left\{\Delta t \log \left(\frac{1}{\Delta t}\right)\right\}^{1 / 2}+\lambda^{1-\alpha}\right], \quad \forall \alpha>0,
$$

for small $\Delta t \leqslant \lambda$, which may indicate that in practical simulation schemes, for fixed $\Delta t$, it is preferable to choose $\lambda$ as small as possible. For $\lambda=(\Delta t)^{1 / 2}, \mathrm{E}\left\|\xi_{\lambda}^{\Delta t}-\xi\right\|_{T}^{2}$ is $\mathrm{O}\left(\Delta t^{1 / 2-\alpha}\right)$, $\forall \alpha>0$ and is $\mathrm{O}\left[\left\{\Delta t \log (1 / \Delta t\}^{1 / 2}\right]\right.$ for $\lambda=\Delta t$. However, if $\lambda<\Delta t$, then the $\beta_{\lambda}\left(\xi_{\lambda}^{\Delta t}\left(t_{k-1}\right)\right) \Delta t$ term may push $\xi_{\lambda}^{\Delta t}$ inwards too much for $\odot$ (see Section 4 ).

Consider the iterative projection scheme.

$$
P^{\Delta t}\left(t_{k}\right)=\Pi\left(P^{\Delta t}\left(t_{k-1}\right)+b\left(P^{\Delta t}\left(t_{k-1}\right)\right) \Delta t_{k}+\sigma\left(P^{\Delta t}\left(t_{k-1}\right)\right) \Delta B_{k}\right),
$$

with $P^{\Delta t}(t)=P^{\Delta t}\left(t_{k-1}\right)$ for $t \in\left[t_{k-1}\right), t_{k}$, and $\Delta t_{k} \leqslant \Delta t$. Under Condition B for $\bar{\odot}$ of Tanaka (1979), and if $\sigma$ is bounded,

$$
\mathrm{E}\left\|P^{\Delta t}-\xi\right\|_{T}^{2}=\mathrm{O}\left[\left\{\Delta t \log \left(\frac{1}{\Delta t}\right)\right\}^{1 / 2}\right],
$$

which can be seen by a slight modification of Theorem 2 of Słomiński (1994) and using

$$
\mathrm{E} \sup _{0 \leqslant s<t \leqslant T,|t-s| \leqslant \Delta t}|B(t)-B(s)|^{2}=\mathrm{O}\left\{\Delta t \log \left(\frac{1}{\Delta t}\right)\right\}
$$


for small $\Delta t$ (see, for example, Pettersson 1995). If $\overline{\mathscr{C}}$ is a convex polyhedron, E\| $P^{\Delta t}-\xi \|_{T}^{2}$ is $\mathrm{O}\{\Delta t \log (1 / \Delta t)\}$ (Pettersson 1995). Observe that (10) can be seen as a variant of the Euler scheme (7):

$$
\begin{aligned}
P^{\Delta t}\left(t_{k}\right)= & P^{\Delta t}\left(t_{k-1}\right)+b\left(P^{\Delta t}\left(t_{k-1}\right)\right) \Delta t_{k}+\sigma\left(P^{\Delta t}\left(t_{k-1}\right)\right) \Delta B_{k} \\
& -\beta_{\lambda}\left(P^{\Delta t}\left(t_{k-1}\right)+b\left(P^{\Delta t}\left(t_{k-1}\right)\right) \Delta t_{k}+\sigma\left(P^{\Delta t}\left(t_{k-1}\right)\right) \Delta B_{k}\right) \Delta t_{k},
\end{aligned}
$$

where $\lambda=\Delta t_{k}$. In order to keep the proof of Theorem 3.1 to the essentials, we concentrate on the case when $b \equiv 0$ and $\sigma$ is a constant matrix. We also let $\Delta t_{k} \equiv \Delta t$. We thus have

$$
\begin{gathered}
\mathrm{d} \xi(t)=\sigma \mathrm{d} B(t)-\mathrm{d} \eta(t), \quad \xi(0)=x_{0}, \\
\mathrm{~d} \xi_{\lambda}(t)=\sigma \mathrm{d} B(t)-\beta_{\lambda}\left(\xi_{\lambda}(t)\right) \mathrm{d} t, \quad \xi_{\lambda}(0)=x_{0}, \\
\xi_{\lambda}^{\Delta t}\left(t_{k}\right)=\xi_{\lambda}^{\Delta t}\left(t_{k-1}\right)+\sigma \Delta B_{k}-\beta_{\lambda}\left(\xi_{\lambda}^{\Delta t}\left(t_{k-1}\right)\right) \Delta t, \quad \xi_{\lambda}^{\Delta t}(0)=x_{0} .
\end{gathered}
$$

Lemma 3.2. Let $\xi_{\lambda}$ be the solution to (14). Then

$$
\sup _{\lambda>0} \mathrm{E}\left\|\xi_{\lambda}\right\|_{T}^{2}<\infty
$$

and

$$
\sup _{\lambda>0} \mathrm{E}\left\{\left(\int_{0}^{T}\left|\beta_{\lambda}\left(\xi_{\lambda}(s)\right)\right| \mathrm{d} s\right)^{2}\right\}<\infty .
$$

Proof. Lemma 3.2 has been proved by Menaldi (1983). However, we show the ideas behind it. For fixed $\lambda>0$, let $\tau_{n}=\inf \left\{t \in[0, T]:\left|\xi_{\lambda}(t)\right| \geqslant n\right\}$ (=T if the set is empty). By Itô's formula

$$
\begin{aligned}
\left|\xi_{\lambda}\left(t \wedge \tau_{n}\right)-x_{0}\right|^{2}= & -2 \int_{0}^{t \wedge \tau_{n}}\left\langle\xi_{\lambda}(s)-x_{0}, \beta_{\lambda}\left(\xi_{\lambda}(s)\right)\right\rangle \mathrm{d} s+2 \int_{0}^{t \wedge \tau_{n}}\left\langle\xi_{\lambda}(s)-x_{0}, \sigma \mathrm{d} B(s)\right\rangle \\
& +|\sigma|^{2}\left(t \wedge \tau_{n}\right) .
\end{aligned}
$$

The first integral is, by (3), less than or equal to zero. By the Burkholder-Davis-Gundy inequality and the inequality $2 a b \leqslant \epsilon a^{2}+b^{2} / \epsilon$, for fixed $t$,

$$
\begin{aligned}
\mathrm{E} \sup _{0 \leqslant s \leqslant t} \int_{0}^{s \wedge \tau_{n}}\left\langle\xi_{\lambda}(s)-x_{0}, \sigma \mathrm{d} B(s)\right\rangle & \leqslant 3 \mathrm{E}\left\{\sup _{0 \leqslant s \leqslant t}\left|\xi_{\lambda}\left(s \wedge \tau_{n}\right)-x_{0}\right|\left(\int_{0}^{t \wedge \tau_{n}}|\sigma|^{2} \mathrm{~d} s\right)^{1 / 2}\right\} \\
& \leqslant \frac{1}{2} \mathrm{E} \sup _{0 \leqslant s \leqslant t}\left|\xi_{\lambda}\left(s \wedge \tau_{n}\right)-x_{0}\right|^{2}+18|\sigma|^{2} T .
\end{aligned}
$$

By a Bellman-Gronwall argument it now follows that $\operatorname{Esup}_{0 \leqslant s \leqslant t}\left|\xi_{\lambda}\left(s \wedge \tau_{n}\right)-x_{0}\right|^{2}$ is uniformly bounded over all $n$ and $\lambda$. Fatou's lemma then gives (16). 
By (1) and (18),

$$
2 \gamma \int_{0}^{t \wedge \tau_{n}} \mid \beta_{\lambda}\left(\xi_{\lambda}(s) \mathrm{d} s \leqslant 2 \int_{0}^{t \wedge \tau_{n}}\left\langle\xi_{\lambda}(s)-x_{0}, \sigma \mathrm{d} B(s)\right\rangle+|\sigma|^{2}\left(t \wedge \tau_{n}\right)-\left|\xi_{\lambda}\left(t \wedge \tau_{n}\right)-x_{0}\right|^{2} .\right.
$$

Itô isomorphism and (16) then give (17).

Now we give a discrete version of Lemma 3.2.

Lemma 3.3. Let $\xi_{\lambda}^{\Delta t}$ be given by (15). Then

$$
\begin{gathered}
\sup _{0<\Delta t \leqslant \lambda} \mathrm{E}\left\|\xi_{\lambda}^{\Delta t}\right\|_{T}^{2}<\infty, \\
\sup _{0<\Delta t \leqslant \lambda^{t_{k}} \leqslant T}\left|\beta_{\lambda}\left(\xi_{\lambda}^{\Delta t}\left(t_{k-1}\right)\right)\right| \Delta t \leqslant \max _{t_{k} \leqslant T}\left|\sigma \Delta B_{k}\right|, \\
\sup _{0<\Delta t \leqslant \lambda} \sum_{t_{k} \leqslant T}\left|\beta_{\lambda}\left(\xi_{\lambda}^{\Delta t}\left(t_{k-1}\right)\right)\right|^{2} \Delta t^{2} \leqslant \sum_{t_{k} \leqslant T}\left|\sigma \Delta B_{k}\right|^{2}, \\
\sup _{0<\Delta t \leqslant \lambda} \mathrm{E}\left\{\left(\sum_{t_{k} \leqslant T}\left|\beta_{\lambda}\left(\xi_{\lambda}^{\Delta t}\left(t_{k-1}\right)\right)\right| \Delta t\right)^{2}\right\}<\infty,
\end{gathered}
$$

where the supremum is over all $\Delta t, \lambda$ such that $0<\Delta t \leqslant \lambda$.

Proof. We first show (19). Evidently,

$$
\begin{aligned}
\left|\xi_{\lambda}^{\Delta t}\left(t_{k}\right)-x_{0}\right|^{2}= & \left|\xi_{\lambda}^{\Delta t}\left(t_{k-1}\right)-x_{0}\right|^{2}+\left|\sigma \Delta B_{k}\right|^{2}+\left|\beta_{\lambda}\left(\xi_{\lambda}^{\Delta t}\left(t_{k-1}\right)\right)\right|^{2} \Delta t^{2} \\
& +2\left\langle\xi_{\lambda}^{\Delta t}\left(t_{k-1}\right)-x_{0}, \sigma \Delta B_{k}\right\rangle-2\left\langle\xi_{\lambda}^{\Delta t}\left(t_{k-1}\right)-x_{0}, \beta_{\lambda}\left(\xi_{\lambda}^{\Delta t}\left(t_{k-1}\right)\right)\right\rangle \Delta t \\
& -2\left\langle\sigma \Delta B_{k}, \beta_{\lambda}\left(\xi_{\lambda}^{\Delta t}\left(t_{k-1}\right)\right)\right\rangle \Delta t .
\end{aligned}
$$

For $\Delta t \leqslant \lambda$, we get, by (3),

$$
\begin{aligned}
\left|\xi_{\lambda}^{\Delta t}\left(t_{k}\right)-x_{0}\right|^{2} \leqslant & \sum_{t_{k} \leqslant T}\left|\sigma \Delta B_{k}\right|^{2}+2 \sum_{t_{k} \leqslant T}\left\langle\xi_{\lambda}^{\Delta t}\left(t_{k-1}\right)-x_{0}, \sigma \Delta B_{k}\right\rangle \\
& -2 \sum_{t_{k} \leqslant T}\left\langle\sigma \Delta B_{k}, \beta_{\lambda}\left(\xi_{\lambda}^{\Delta t}\left(t_{k-1}\right)\right)\right\rangle \Delta t
\end{aligned}
$$

which, since $\beta_{\lambda}\left(\xi_{\lambda}^{\Delta t}\left(t_{k-1}\right)\right)$ is $\mathscr{F}_{t_{k-1}}$ adapted yields

$$
\mathrm{E}\left|\xi_{\lambda}^{\Delta t}\left(t_{k}\right)-x_{0}\right|^{2} \leqslant|\sigma|^{2} t_{k} \leqslant|\sigma|^{2} T
$$

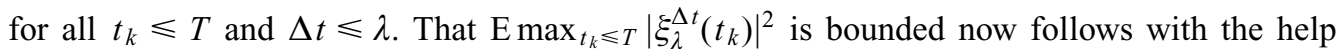
of the Burkholder-Davis-Gundy inequality. We have, for example, 
$2 \mathrm{E} \max _{t_{k} \leqslant T} \sum_{t_{j} \leqslant t_{k}}\left\langle\sigma \Delta B_{j}, \beta_{\lambda}\left(\xi_{\lambda}^{\Delta t}\left(t_{j-1}\right)\right)\right\rangle \Delta t \leqslant 6 \mathrm{E}\left\{\left(\sum_{t_{k} \leqslant T}\left|\beta_{\lambda}\left(\xi_{\lambda}^{\Delta t}\left(t_{k-1}\right)\right) \Delta t\right|^{2}|\sigma|^{2} \Delta t\right)^{1 / 2}\right\}$

which, since $\Delta t \leqslant \lambda$ and $|\beta(x)| \leqslant\left|x-x_{0}\right|$ for all $x$ in $\mathfrak{R}^{d}$, is dominated by

$$
6 \mathrm{E}\left\{\left(\sum_{t_{k} \leqslant T}\left|\xi_{\lambda}^{\Delta t}\left(t_{k-1}\right)-x_{0}\right|^{2}|\sigma|^{2} \Delta t\right)^{1 / 2}\right\} \leqslant 3|\sigma|^{2}+3 \sum_{t_{k} \leqslant T} \mathrm{E}\left|\xi_{\lambda}^{\Delta t}\left(t_{k-1}\right)-x_{0}\right|^{2} \Delta t .
$$

The assertion (19) then follows trivially.

Now we prove (20). Note that

$$
\xi_{\lambda}^{\Delta t}\left(t_{k}\right)=\Pi\left(\xi_{\lambda}^{\Delta t}\left(t_{k-1}\right)\right)+\sigma \Delta B_{k}+\left(1-\frac{\Delta t}{\lambda}\right) \beta\left(\xi_{\lambda}^{\Delta t}\left(t_{k-1}\right)\right)
$$

hence, by the definition of $\Pi$ and $\beta$,

$$
\begin{aligned}
\left|\beta\left(\xi_{\lambda}^{\Delta t}\left(t_{k}\right)\right)\right| & =\left|\xi_{\lambda}^{\Delta t}\left(t_{k}\right)-\Pi\left(\xi_{\lambda}^{\Delta t}\left(t_{k}\right)\right)\right| \\
& \leqslant\left|\xi_{\lambda}^{\Delta t}\left(t_{k}\right)-\Pi\left(\xi_{\lambda}^{\Delta t}\left(t_{k-1}\right)\right)\right| \\
& \leqslant\left|\sigma \Delta B_{k}\right|+\left(1-\frac{\Delta t}{\lambda}\right)\left|\beta\left(\xi_{\lambda}^{\Delta t}\left(t_{k-1}\right)\right)\right|
\end{aligned}
$$

and, consequently,

$$
\max _{t_{k} \leqslant T}\left|\beta\left(\xi_{\lambda}^{\Delta t}\left(t_{k}\right)\right)\right| \leqslant \max _{t_{k} \leqslant T}\left|\sigma \Delta B_{k}\right|+\left(1-\frac{\Delta t}{\lambda}\right) \max _{t_{k} \leqslant T}\left|\beta\left(\xi_{\lambda}^{\Delta t}\left(t_{k-1}\right)\right)\right|,
$$

which gives (20).

If $\Delta t=\lambda$, then (21) follows immediately by (25). Else, squaring of (25) gives

$$
\begin{aligned}
\left|\beta\left(\xi_{\lambda}^{\Delta t}\left(t_{k}\right)\right)\right|^{2} & \leqslant\left|\sigma \Delta B_{k}\right|^{2}+\left(1-\frac{\Delta t}{\lambda}\right)^{2}\left|\beta\left(\xi_{\lambda}^{\Delta t}\left(t_{k-1}\right)\right)\right|^{2}+2\left(1-\frac{\Delta t}{\lambda}\right)\left|\beta\left(\xi_{\lambda}^{\Delta t}\left(t_{k-1}\right)\right)\right|\left|\sigma \Delta B_{k}\right| \\
& \leqslant\left(1-\frac{\Delta t}{\lambda}\right)^{2}(1+\epsilon)\left|\beta\left(\xi_{\lambda}^{\Delta t}\left(t_{k-1}\right)\right)\right|^{2}+\left(1+\frac{1}{\epsilon}\right)\left|\sigma \Delta B_{k}\right|^{2} \\
& =\left(1-\frac{\Delta t}{\lambda}\right)^{2}\left|\beta\left(\xi_{\lambda}^{\Delta t}\left(t_{k-1}\right)\right)\right|^{2}+\frac{\lambda}{\Delta t}\left|\sigma \Delta B_{k}\right|^{2} \\
\epsilon & =\frac{\Delta t / \lambda}{1-\Delta t / \lambda} .
\end{aligned}
$$


Hence,

$$
\begin{aligned}
\sum_{t_{k} \leqslant T}\left|\beta\left(\xi_{\lambda}^{\Delta t}\left(t_{k-1}\right)\right)\right|^{2} & \leqslant \sum_{t_{k} \leqslant T}\left|\beta\left(\xi_{\lambda}^{\Delta t}\left(t_{k}\right)\right)\right|^{2} \\
& \leqslant \sum_{t_{k} \leqslant T}\left(1-\frac{\Delta t}{\lambda}\right)^{2}\left|\beta\left(\xi_{\lambda}^{\Delta t}\left(t_{k-1}\right)\right)\right|^{2}+\sum_{t_{k} \leqslant T} \frac{\lambda}{\Delta t}\left|\sigma \Delta B_{k}\right|^{2}
\end{aligned}
$$

i.e.,

$$
\sum_{t_{k} \leqslant T} \frac{\left|\beta\left(\xi_{\lambda}^{\Delta t}\left(t_{k-1}\right)\right)\right|^{2} \Delta t^{2}}{\lambda^{2}} \leqslant \sum_{t_{k} \leqslant T}\left|\sigma \Delta B_{k}\right|^{2} .
$$

Finally, we obtain (22). By (1) and (23),

$$
\begin{aligned}
2 \gamma\left|\beta_{\lambda}\left(\xi_{\lambda}^{\Delta t}\left(t_{k-1}\right)\right)\right| \Delta t \leqslant & \left|\xi_{\lambda}^{\Delta t}\left(t_{k-1}\right)-x_{0}\right|^{2}-\left|\xi_{\lambda}^{\Delta t}\left(t_{k}\right)-x_{0}\right|^{2}+\left|\sigma \Delta B_{k}\right|^{2}+\left|\beta_{\lambda}\left(\xi_{\lambda}^{\Delta t}\left(t_{k-1}\right)\right)\right|^{2} \Delta t^{2} \\
& +2\left\langle\xi_{\lambda}^{\Delta t}\left(t_{k-1}\right)-x_{0}, \sigma \Delta B_{k}\right\rangle-2\left\langle\sigma \Delta B_{k}, \beta_{\lambda} \mid\left(\xi_{\lambda}^{\Delta t}\left(t_{k-1}\right)\right)\right\rangle \Delta t .
\end{aligned}
$$

Thus,

$$
\begin{aligned}
2 \gamma \sum_{t_{k} \leqslant T}\left|\beta_{\lambda}\left(\xi_{\lambda}^{\Delta t}\left(t_{k-1}\right)\right)\right| \Delta t \leqslant & \sum_{t_{k} \leqslant T}\left|\sigma \Delta B_{k}\right|^{2}+\sum_{t_{k} \leqslant T}\left|\beta_{\lambda}\left(\xi_{\lambda}^{\Delta t}\left(t_{k-1}\right)\right)\right|^{2} \Delta t^{2} \\
& +2 \sum_{t_{k} \leqslant T}\left\langle\xi_{\lambda}^{\Delta t}\left(t_{k-1}\right)-x_{0}, \sigma \Delta B_{k}\right\rangle \\
& -2 \sum_{t_{k} \leqslant T}\left\langle\sigma \Delta B_{k}, \beta_{\lambda}\left(\xi_{\lambda}^{\Delta t}\left(t_{k-1}\right)\right)\right\rangle \Delta t
\end{aligned}
$$

By using (21) we get (22).

\section{Proof of Theorem 3.1.}

(i) $b \equiv 0$ and $\sigma$ constant. Recall that $\xi_{\lambda}^{\Delta t}$ is interpolated:

$$
\xi_{\lambda}^{\Delta t}(t)=x_{0}-\int_{0}^{t} \beta_{\lambda}\left(\xi_{\lambda}^{\Delta t}\left(s^{\Delta t}\right)\right) \mathrm{d} s+\int_{0}^{t} \sigma \mathrm{d} B(s),
$$

where $s^{\Delta t}=\max \left\{t_{k}: t_{k} \leqslant s\right\}$. Then by Itô's formula and (3),

$$
\begin{aligned}
\left|\xi_{\lambda}(t)-\xi_{\lambda}^{\Delta t}(t)\right|^{2} & =-2 \int_{0}^{t}\left\langle\beta_{\lambda}\left(\xi_{\lambda}(s)\right)-\beta_{\lambda}\left(\xi_{\lambda}^{\Delta t}\left(s^{\Delta t}\right)\right), \xi_{\lambda}(s)-\xi_{\lambda}^{\Delta t}(s)\right\rangle \mathrm{d} s \\
& \leqslant-2 \int_{0}^{t}\left\langle\beta_{\lambda}\left(\xi_{\lambda}(s)\right)-\beta_{\lambda}\left(\xi_{\lambda}^{\Delta t}\left(s^{\Delta t}\right)\right), \xi_{\lambda}^{\Delta t}\left(s^{\Delta t}\right)-\xi_{\lambda}^{\Delta t}(s)\right\rangle \mathrm{d} s .
\end{aligned}
$$


Hence

$$
\begin{aligned}
\mathrm{E}\left\|\xi_{\lambda}-\xi_{\lambda}^{\Delta t}\right\|_{T}^{2} \leqslant & 2\left\{\mathrm{E} \sup _{0 \leqslant t \leqslant T} \mid \xi_{\lambda}^{\Delta t}(t)-\xi_{\lambda}^{\Delta t}\left(\left.\left(t^{\Delta t}\right)\right|^{2}\right\}^{1 / 2}\left[\mathrm{E}\left\{\left(\int_{0}^{T}\left|\beta_{\lambda}\left(\xi_{\lambda}(s)\right)\right| \mathrm{d} s\right)^{2}\right\}\right]^{1 / 2}\right. \\
& +2\left\{\operatorname{E}_{0 \leqslant t \leqslant T}\left|\xi_{\lambda}^{\Delta t}(t)-\xi_{\lambda}^{\Delta t}\left(t^{\Delta t}\right)\right|^{2}\right\}^{1 / 2}\left[\mathrm{E}\left\{\left(\sum_{t_{k} \leqslant T}\left|\beta_{\lambda}\left(\xi_{\lambda}^{\Delta t}\left(t_{k-1}\right)\right)\right| \Delta t\right)^{2}\right\}\right]^{1 / 2},
\end{aligned}
$$

where

$$
\begin{aligned}
\left\{\mathrm{E} \sup _{0 \leqslant t \leqslant T}\left|\xi_{\lambda}^{\Delta t}(t)-\xi_{\lambda}^{\Delta t}\left(t^{\Delta t}\right)\right|^{2}\right\}^{1 / 2} \leqslant & |\sigma|\left\{\mathrm{E} \sup _{0 \leqslant t \leqslant T}\left|B(t)-B\left(t^{\Delta t}\right)\right|^{2}\right\}^{1 / 2} \\
& +\left[\mathrm{E}\left\{\max _{t_{k} \leqslant T}\left|\beta_{\lambda}\left(\xi_{\lambda}^{\Delta t}\left(t_{k-1}\right)\right)\right|^{2} \Delta t^{2}\right\}\right]^{1 / 2}
\end{aligned}
$$

(it also works if $\sigma$ is not constant but bounded). The proof is completed by (20), the modulus of continuity (12) of the Brownian motion, and the boundedness results (17) and (22).

(ii) $b$ and $\sigma$ Lipschitz continuous, $\sigma$ bounded. Modify (i) by elementary computations. For example, for a corresponding statement of Lemma 3.3, use that

$$
\begin{aligned}
\left\langle b\left(\xi_{\lambda}^{\Delta t}\left(t_{k-1}\right)\right) \Delta t, \beta_{\lambda}\left(\xi_{\lambda}^{\Delta t}\left(t_{k-1}\right) \Delta t\right\rangle\right. & \leqslant \frac{\left|b\left(\xi_{\lambda}^{\Delta t}\left(t_{k-1}\right)\right)\right|\left|\beta\left(\xi_{\lambda}^{\Delta t}\left(t_{k-1}\right)\right)\right| \Delta t^{2}}{\lambda} \\
& \leqslant\left|b\left(\xi_{\lambda}^{\Delta t}\left(t_{k-1}\right)\right)\right|\left|\beta\left(\xi_{\lambda}^{\Delta t}\left(t_{k-1}\right)\right)\right| \Delta t \\
& \leqslant c\left(1+\left|\xi_{\lambda}^{\Delta t}\left(t_{k-1}\right)\right|^{2}\right) \Delta t,
\end{aligned}
$$

and for calculations corresponding to (i), use inequalities as in Lemma 3.2:

$$
\begin{aligned}
\operatorname{E} \sup _{t \leqslant T}\left|\int_{0}^{t}\left\langle\xi_{\lambda}(s)-\xi_{\lambda}^{\Delta t}(s), \sigma\left(\xi_{\lambda}(s)\right)-\sigma\left(\xi_{\lambda}^{\Delta t}(s)\right) \mathrm{d} B(s)\right\rangle\right| & \leqslant \frac{1}{2} \mathrm{E}\left\|\xi_{\lambda}-\xi_{\lambda}^{\Delta t}\right\|_{T}^{2} \\
& +18 \int_{0}^{T} \mathrm{E}\left|\sigma\left(\xi_{\lambda}(s)\right)-\sigma\left(\xi_{\lambda}^{\Delta t}(s)\right)\right|^{2} \mathrm{~d} s .
\end{aligned}
$$

Remark 3.4. By using a recent generalized result (1) of Storm (1995), we can easily show a bounded variation result for the projection scheme (10) similarly to (22). This is important for proving convergence of the type (11).

For $x \in \partial \odot$ let $\mathscr{N}(x)$ be the outward-directed normal cone at $x$,

$$
\mathscr{N}(x)=\left\{n \in \mathfrak{R}^{d}:-\langle x-y, n\rangle \leqslant 0 \quad \forall y \in \overline{\mathscr{O}}\right\},
$$

and for $x$ in $\odot$ let $\mathscr{N}(x)=0 \in \mathfrak{l}^{d}$. Then, by Proposition 2.2 of Storm (1995), there exists a $\gamma>0$ such that

$$
\gamma|n| \leqslant\left\langle x-x_{0}, n\right\rangle, \quad \forall x \in \overline{\mathscr{O}}, \forall n \in \mathscr{N}(x)
$$


Consider again, for simplicity, the case $b \equiv 0$ and $\sigma$ constant. Then

$$
P^{\Delta t}\left(t_{k}\right)=P^{\Delta t}\left(t_{k-1}\right)+\sigma \Delta B_{k}-\Delta \eta_{k},
$$

where

$$
\Delta \eta_{k}=\beta\left\{P^{\Delta t}\left(t_{k-1}\right)+\sigma \Delta B_{k}\right\} \in \mathscr{N}\left\{\Pi\left(P^{\Delta t}\left(t_{k-1}\right)+\sigma \Delta B_{k}\right)\right\}=\mathscr{N}\left(P^{\Delta t}\left(t_{k}\right)\right) .
$$

Since

$$
\left|P^{\Delta t}\left(t_{k}\right)-x_{0}\right|^{2}=\left|P^{\Delta t}\left(t_{k-1}\right)-x_{0}\right|^{2}+\left|\sigma \Delta B_{k}\right|^{2}-2\left\langle P^{\Delta t}\left(t_{k}\right)-x_{0}, \Delta \eta_{k}\right\rangle-\left|\Delta \eta_{k}\right|^{2},
$$

by (27), (29) and (30), we get

$$
2 \gamma \sum_{t_{k} \leqslant T}\left|\Delta \eta_{k}\right| \leqslant 2 \sum_{t_{k} \leqslant T}\left\langle P^{\Delta t}\left(t_{k}\right)-x_{0}, \Delta \eta_{k}\right\rangle \leqslant \sum_{t_{k} \leqslant T}\left|\sigma \Delta B_{k}\right|^{2}
$$

which gives the version of (22) searched for.

To show convergence of the type (6) and (11) it is also important to use a bounded variation result for $\eta$, where $\eta$ is the bounded variation process in Definition 2.1. Since $\eta$ is the limit of $\int_{0}^{t} \beta_{\lambda}\left(\xi_{\lambda}(s)\right) \mathrm{d} s$ in the mean square sense, uniformly on [0,T] (Menaldi 1983), there exists a sequence $\lambda_{n} \downarrow 0$ such that

$$
\sup _{0 \leqslant t \leqslant T}\left|\eta(t)-\int_{0}^{t} \beta_{\lambda_{n}}\left(\xi_{\lambda_{n}}(s)\right) \mathrm{d} s\right| \stackrel{\text { (a.s.) }}{\rightarrow} 0, \quad \lambda_{n} \downarrow 0 .
$$

This implies that the variation $|\eta|(T)$ of $\eta$ on $[0, T]$ is dominated by $\liminf _{\lambda_{n} \downarrow 0} \int_{0}^{T}\left|\beta_{\lambda_{n}}\left(\xi_{\lambda_{n}}(s)\right)\right| \mathrm{d} s$ (a.s.). Hence, by Fatou's lemma and (17), it follows that $\mathrm{E}\left\{(|\eta|(T))^{2}\right\}<\infty$.

\section{Comparison of $\xi_{\lambda}^{\delta}$ and $\mathrm{P}^{\delta}$}

We now compare the methods (7) and (10). Note that, if $\Delta t=\lambda, b \equiv 0$ and $\sigma$ is constant,

$$
P^{\Delta t}\left(t_{k}\right)=\Pi\left(\xi_{\Delta t}^{\Delta t}\left(t_{k}\right), \quad \xi_{\Delta t}^{\Delta t}\left(t_{k}\right)=P^{\Delta t}\left(t_{k-1}\right)+\sigma \Delta B_{k},\right.
$$

and, by using (26), (28) and (29),

$$
\left|P^{\Delta t}\left(t_{k}\right)-P^{\Delta t}\left(t_{k-1}\right)\right| \leqslant\left|\sigma \Delta B_{k}\right| .
$$

We especially have

$$
P^{\Delta t}\left(t_{k}\right)-\Pi\left(\xi_{\Delta t}^{\Delta t}\left(t_{k}\right)\right)=0, \quad \max _{t_{k} \leqslant T}\left|P^{\Delta t}\left(t_{k}\right)-\xi_{\Delta t}^{\Delta t}\left(t_{k}\right)\right| \leqslant \max _{t_{k} \leqslant T}\left|\sigma \Delta B_{k}\right| .
$$

In one dimension, with $b \equiv 0, m=d=1, \sigma \equiv 1, x_{0}=B(0)=0$ and $\mathscr{Q}=(0, \infty)$, the solution $(\xi, \eta)$ to $\operatorname{RSDE}(13)$, is well known to be given by

$$
\xi(t)=B(t)-\min _{0 \leqslant s \leqslant t} B(s), \quad \eta(t)=\min _{0 \leqslant s \leqslant t} B(s) .
$$


Further, it is easy to show for fixed $\Delta t$ that, if $\lambda \geqslant \Delta t$,

$$
\xi_{\lambda}^{\Delta t}\left(t_{k}\right) \leqslant \xi_{\Delta t}^{\Delta t}\left(t_{k}\right) \leqslant \max \left(\xi_{\Delta t}^{\Delta t}\left(t_{k}\right), 0\right)=P^{\Delta t}\left(t_{k}\right)=B\left(t_{k}\right)-\min _{0 \leqslant t_{j} \leqslant t_{k}} B\left(t_{j}\right) \leqslant \xi\left(t_{k}\right),
$$

which means that, in at least this case, it is appropriate to choose $\lambda \leqslant \Delta t$ when simulating $\xi_{\lambda}^{\Delta t}\left(t_{k}\right)$. However, if $\lambda<\Delta t$, then Lemma 3.3 cannot be used. Instead we note the following. Let

$$
q_{\lambda}^{\Delta t}=\frac{\mathrm{E} \xi_{\lambda}^{\Delta t}\left(t_{2}\right)}{\mathrm{E} \xi\left(t_{2}\right)}=\frac{\mathrm{E}\left[B\left(t_{2}\right)-\min \left\{B\left(t_{1}\right), 0\right\} \Delta t / \lambda\right]}{\mathrm{E}\left[B\left(t_{2}\right)-\min \left\{(B(s), 0): s \leqslant t_{2}\right\}\right]}=\frac{\Delta t / \lambda}{2^{3 / 2}} .
$$

If for example for some $\epsilon>0, \lambda=\Delta t^{1+\epsilon}(<\Delta t)$, then $q_{\lambda}^{\Delta t} \rightarrow \infty$ as $\Delta t \downarrow 0$, which means that the penalty term $\beta_{\lambda}\left(\xi_{\lambda}^{\Delta t}\left(t_{k-1}\right) \Delta t\right.$ pushes $\xi_{\lambda}^{\Delta t}\left(t_{k}\right)$ inwards too much $(0, \infty)$. In particular, if $\epsilon>\frac{1}{2}$, then

$$
\left(\mathrm{E} \max _{t_{k} \leqslant T}\left\|\xi_{\lambda}^{\Delta t}-\xi\right\|_{T}^{2}\right)^{1 / 2} \geqslant\left(q_{\lambda}^{\Delta t}-1\right) \mathrm{E} \xi\left(t_{2}\right) \rightarrow \infty, \quad \text { as } \Delta t \rightarrow 0 .
$$

Since $\xi_{\lambda}^{\Delta t}$ above zero follows the path of the Brownian motion, the penalty term, when it is not zero, is generically of order $(\Delta t)^{3 / 2} / \lambda$ which, if $\lambda=\Delta t^{1+\epsilon}, \epsilon>0$, creates too large jumps upwards compared with the increments of $\xi$ which are of order $(\Delta t)^{1 / 2}$. This behaviour of $\xi_{\lambda}^{\Delta t}$ also holds in higher dimensions if $\mathscr{Q}$ is a half-space. If $\mathscr{O}$ is bounded and convex with $C^{1}$ boundary, a similar result also seems plausible by a localization argument.

Here is a generalized result of (31) when $b$ may not be 0 and $\sigma$ may not be a constant.

Proposition 4.1. Let $\xi_{\lambda}^{\Delta t}$ be given by (7), where $\Delta t=\lambda$, and $P^{\Delta t}$ by (10). Assume that $b$ and $\sigma$ satisfy the usual Lipschitz conditions and $\sigma$ is bounded. Then

$$
\begin{gathered}
\mathrm{E} \max _{t_{k} \leqslant T}\left|P^{\Delta t}\left(t_{k}\right)-\Pi\left(\xi_{\Delta t}^{\Delta t}\right)\left(t_{k}\right)\right|^{2}=O(\Delta t), \\
\mathrm{E} \max _{t_{k} \leqslant T}\left|P^{\Delta t}\left(t_{k}\right)-\xi_{\Delta t}^{\Delta t}\left(t_{k}\right)\right|^{2}=O\left\{\Delta t \log \left(\frac{1}{\Delta t}\right)\right\},
\end{gathered}
$$

for small $\Delta t$.

Proof. In order to keep to essentials, we also here consider the case when $b \equiv 0$. We can write

$$
P^{\Delta t}\left(t_{k}\right)=P^{\Delta t}\left(t_{k-1}\right)+\sigma\left(P^{\Delta t}\left(t_{k-1}\right)\right) \Delta B_{k}-\Delta \eta_{k}, \quad \Delta \eta_{k} \in \mathscr{N}\left(P^{\Delta t}\left(t_{k}\right)\right)
$$

and

$$
\begin{gathered}
\Pi\left(\xi_{\Delta t}^{\Delta t}\left(t_{k}\right)\right)=\Pi\left(\xi_{\Delta t}^{\Delta t}\left(t_{k-1}\right)\right)+\sigma\left(\xi_{\Delta t}^{\Delta t}\left(t_{k-1}\right)\right) \Delta B_{k}+\beta\left(\xi_{\Delta t}^{\Delta t}\left(t_{k}\right)\right), \\
\beta\left(\xi_{\Delta t}^{\Delta t}\left(t_{k}\right)\right) \in \mathscr{N}\left(\Pi\left(\xi_{\Delta t}^{\Delta t}\left(t_{k}\right)\right)\right) .
\end{gathered}
$$

Hence, by careful but elementary calculations, 


$$
\begin{aligned}
\left|P^{\Delta t}\left(t_{k}\right)-\Pi\left(\xi_{\Delta t}^{\Delta t}\left(t_{k}\right)\right)\right|^{2}= & \left|P^{\Delta t}\left(t_{k-1}\right)-\Pi\left(\xi_{\Delta t}^{\Delta t}\left(t_{k-1}\right)\right)\right|^{2}+\left|\left\{\sigma\left(P^{\Delta t}\left(t_{k-1}\right)\right)-\sigma\left(\xi_{\Delta t}^{\Delta t}\left(t_{k-1}\right)\right)\right\} \Delta B_{k}\right|^{2} \\
& -2\left\langle P^{\Delta t}\left(t_{k-1}\right)-\Pi\left(\xi_{\Delta t}^{\Delta t}\left(t_{k-1}\right)\right),\left\{\sigma\left(P^{\Delta t}\left(t_{k-1}\right)\right)-\sigma\left(\xi_{\Delta t}^{\Delta t}\left(t_{k-1}\right)\right)\right\} \Delta B_{k}\right\rangle \\
& -2\left\langle P^{\Delta t}\left(t_{k}\right)-\Pi\left(\xi_{\Delta t}^{\Delta t}\left(t_{k}\right)\right), \Delta \eta_{k}-\beta\left(\xi_{\Delta t}^{\Delta t}\left(t_{k}\right)\right)\right\rangle-\left|\Delta \eta_{k}-\beta\left(\xi_{\Delta t}^{\Delta t}\left(t_{k}\right)\right)\right|^{2} .
\end{aligned}
$$

By (34), (35) and (26),

$$
-2\left\langle P^{\Delta t}\left(t_{k}\right)-\Pi\left(\xi_{\Delta t}^{\Delta t}\left(t_{k}\right)\right), \Delta \eta_{k}-\beta\left(\xi_{\Delta t}^{\Delta t}\left(t_{k}\right)\right)\right\rangle \leqslant 0 .
$$

Consequently,

$$
\begin{aligned}
& \left|P^{\Delta t}\left(t_{k}\right)-\Pi\left(\xi_{\Delta t}^{\Delta t}\left(t_{k}\right)\right)\right|^{2} \leqslant \\
& \quad-2 \sum_{t_{j} \leqslant t_{k}}\left\langle P^{\Delta t}\left(t_{j-1}\right)-\Pi\left(\xi_{\Delta t}^{\Delta t}\left(t_{j-1}\right)\right),\left\{\sigma\left(P^{\Delta t}\left(t_{j-1}\right)\right)-\sigma\left(\xi_{\Delta t}^{\Delta t}\left(t_{j-1}\right)\right)\right\} \Delta B_{j}\right\rangle \\
& \quad+\sum_{t_{j} \leqslant t_{k}} \mid\left\{\left.\sigma\left(P^{\Delta t}\left(t_{j-1}\right)-\sigma\left(\xi_{\Delta t}^{\Delta t}\left(t_{j-1}\right)\right)\right\} \Delta B_{k}\right|^{2} .\right.
\end{aligned}
$$

For fixed $t_{1}$, by the Burkholder-Davis-Gundy inequality,

$$
\begin{aligned}
& \mathrm{E} \max _{t_{k} \leqslant t_{l}}\left(-2 \sum_{t_{j} \leqslant t_{k}}\left\langle P^{\Delta t}\left(t_{j-1}\right)-\Pi\left(\xi_{\Delta t}^{\Delta t}\left(t_{j-1}\right), \sigma\left(P^{\Delta t}\left(t_{j-1}\right)\right)-\sigma\left(\xi_{\Delta t}^{\Delta t}\left(t_{j-1}\right)\right)\right\} \Delta B_{j}\right\rangle\right) \\
& \left.\quad \leqslant 6 \mathrm{E} \max _{t_{k} \leqslant t_{l}}\left|P^{\Delta t}\left(t_{k-1}\right)-\Pi\left(\xi_{\Delta t}^{\Delta t}\left(t_{k-1}\right)\right)\right|\left(\sum_{t_{k} \leqslant t_{l}} \mid \sigma\left(P^{\Delta t}\left(t_{k-1}\right)\right)\right)-\left.\sigma\left(\xi_{\Delta t}^{\Delta t}\left(t_{j-1}\right)\right)\right|^{2} \Delta t\right)^{1 / 2} \\
& \quad \leqslant \frac{1}{2} \mathrm{E} \max _{t_{k} \leqslant t_{l}}\left|P^{\Delta t}\left(t_{k-1}\right)-\Pi\left(\xi_{\Delta t}^{\Delta t}\left(t_{k-1}\right)\right)\right|^{2}+18 \mathrm{E} \sum_{t_{k} \leqslant t_{l}}\left|\sigma\left(P^{\Delta t}\left(t_{k-1}\right)\right)-\sigma\left(\xi_{\Delta t}^{\Delta t}\left(t_{k-1}\right)\right)\right|^{2} \Delta t .
\end{aligned}
$$

Hence

$$
\mathrm{E} \max _{t_{k} \leqslant t_{l}}\left|P^{\Delta t}\left(t_{k}\right)-\Pi\left(\xi_{\Delta t}^{\Delta t}\left(t_{k}\right)\right)\right|^{2} \leqslant 38 \sum_{t_{k} \leqslant t_{l}} \mathrm{E}\left|\sigma\left(P^{\Delta t}\left(t_{k-1}\right)\right)-\sigma\left(\xi_{\Delta t}^{\Delta t}\left(t_{k-1}\right)\right)\right|^{2} \Delta t
$$

By a corresponding inequality to (25), it is readily shown that

$$
\left|\xi_{\Delta t}^{\Delta t}\left(t_{k}\right)-\Pi\left(\xi_{\Delta t}^{\Delta t}\left(t_{k}\right)\right)\right| \leqslant\left|\sigma\left(\xi_{\Delta t}^{\Delta t}\left(t_{k-1}\right)\right) \Delta B_{k}\right| .
$$

Hence, by the Lipschitz assumption of $\sigma$, a generalization of (19), (36) and (37),

$$
\mathrm{E} \max _{t_{k} \leqslant t_{l}}\left|P^{\Delta t}\left(t_{k}\right)-\Pi\left(\xi_{\Delta t}^{\Delta t}\right)\left(t_{k}\right)\right|^{2} \leqslant c \sum_{t_{k} \leqslant t_{l}} \mathrm{E}\left|P\left(t_{k-1}\right)-\Pi\left(\xi_{\Delta t}^{\Delta t}\left(t_{k-1}\right)\right)\right|^{2} \Delta t+c \sum_{t_{k} \leqslant t_{l}} \mathrm{E}\left|\Delta B_{k}\right|^{2} \Delta t
$$

which gives (32) by a discrete version of the Bellman-Gronwall inequality.

By (32), (37) and (12), the claim (33) is proved (Condition B of Tanaka 1979 is not needed). 


\section{Acknowledgement}

The major part of this work was done while visiting the Center for Stochastic Processes, Chapel Hill, NC, USA. The work was supported by the Swedish Research Council for Engineering Sciences, Grant 282-94-971 and the Office of Naval Research Grant N00014-931-0043 and Grant N00014-93-1-0841.

\section{References}

Asmussen, S. (1992) Queueing simulation in heavy traffic. Math. Oper. Res., 17, 84-111.

Aubin, J.P. and Cellina, A. (1984) Differential Inclusions. Berlin: Springer-Verlag.

Krée, P. and Soize, C. (1986) Mathematics of Random Phenomena. Dordrecht: Reidel.

Lions, P.-L., Menaldi, J.-L. and Snitzman A.-S. (1981) Construction de processus de diffusion réfléchis par pénalisation du domaine. C.R. Acad. Sci. Paris, Ser. 1, 292, 559-562.

Liu, Y. (1993) Numerical approaches to stochastic differential equations with boundary conditions. Thesis, Purdue University.

Menaldi, J.-L. (1983) Stochastic variational inequality for reflected diffusion. Indiana Univ. Math. J. 32, $733-744$.

Pettersson, R. (1995) Approximations for stochastic differential equation with reflecting convex boundaries. Stochastic Proc. Appl., 59, 295-308.

Shepp, L.A. and Shiryaev, A.N. (1994) A new look at pricing of the "Russian option". Theory Probab. Appl., 39, 103-119.

Słomiński, L. (1994) On approximation of solutions of multidimensional SDE's with reflecting boundary conditions. Stochastic Proc. Appl., 50, 197-219.

Storm, A. (1995) Stochastic differential equations with a convex constraint. Stochastics, 53, 241-274.

Tanaka, H. (1979) Stochastic differential equations with reflecting boundary condition in convex regions. Hiroshima Math. J., 9, 163-177.

Received January 1996 and revised September 1996 